FROM: Weber DJ, Rutala WA. Risks and prevention of nosocomial transmission of rare zoonotic diseases. Clin Infect Dis 2001;32:446-456.

\section{Efficacy of Antiseptics Tested on Skin}

Messager and colleagues from the Welsh School of Pharmacy, Cardiff University, United Kingdom, reported on an ex vivo test used to determine efficacy of germicides used on skin. While there are many skin antiseptics commercially available and their antibacterial activity has often been well studied, their potential effectiveness on skin remains poorly documented. To date, in vivo protocols designed for the testing of the antimicrobial efficacy of antiseptics cannot use, for ethical reasons, pathogenic bacteria or new formulations whose toxicity in human subjects is unknown. An ex vivo test recently was developed to overcome these problems. Freshly excised human skin from abdominal or breast reduction was placed in a diffusion cell containing a maintenance medium in the recipient compartment. A bacterial inoculum was then applied to the stratum corneum and, after a drying step, antiseptic formulations were evaluated for their antimicrobial activity.

Several microorganisms were investigated (Staphylococcus aureus, methicillin-resistant $S$ aureus (MRSA), Enterococcus faecalis, vancomycin-resistant Enterococcus faecium (VRE), Staphylococcus epidermidis, Pseudomonas aeruginosa, and Escherichia coli), along with several biocides (para-chloro-meta-xylenol [PCMX], active compound of Dettol; povidone iodine; triclosan (in isopropanol); and chlorhexidine. Results from the ex vivo test were compared with results obtained in suspension and glass-carrier tests. The bactericidal activity of the biocides depended upon the test performed, and results generally were significantly different from one method to the other. All biocides tested in the suspension test achieved $>4-\log _{10}$ reduction in viable bacterial concentrations, apart from povidone iodine tested against $E$ faecalis and VRE. The antibacterial activity of biocides tested in the glasscarrier test was significantly lower than in the suspension test, with the exception of triclosan in isopropanol, which was as effective in both suspension and glass-carrier test. In the ex vivo test, triclosan in isopropanol achieved a $\log _{10}$ reduction in viable bacterial concentration of 1.105 to 1.771 (with the exception of $P$ aeruginosa, with $0.758-\log _{10}$ reduction). PCMX, povidone iodine, and chlorhexidine achieved $\log _{10}$ reductions in viable bacterial concentration of 0.303 to 0.901 . Chlorhexidine tested against $P$ aeruginos $a$ produced a $1.94-\log _{10}$ reduction in concentration.

These results confirm previous observations about the need for testing the antimicrobial activity of antiseptics on skin surface to determine their in situ efficacy and encourage further the use of the ex vivo protocol.

FROM: Messager S, Goddard PA, Dettmar PW, Maillard JY. Determination of the antibacterial efficacy of several antiseptics tested on skin by an 'ex vivo' test. $J$ Med Microbiol 2001;50:284-292.

\section{VRE in Stools Submitted for $C$ difficile Testing}

Hacek and coinvestigators from Northwestern University Medical Center, Chicago, investigated a method for screening vancomycin-resistant enterococci (VRE) that used specimens submitted for Clostridium difficile testing. They compared this approach to the focused surveillance program of high-risk units during October 1997 to compare the yield of VRE and multidrug-resistant Enterobacteriaceae (MDRE) with both methods. Of the stools submitted for $C$ difficile testing, $14 \%$ were positive for VRE or MDRE, whereas rectal swabs from routine surveillance yielded 11\% VRE- or MDRE-positive results. Although stools submitted for $C$ difficile testing resulted in a higher percentage of positive cultures, $14 \mathrm{VRE}$ - and 2 MDRE-positive patients from their high-risk population were missed because many patients had no stool submitted for $C$ difficile testing.

The authors concluded that, while screening stools submitted for $C$ difficile testing cannot replace their focused surveillance program, it appears advantageous to assess these stools at various intervals to detect new patient reservoirs of drug-resistant organisms that may benefit from routine surveillance cultures.

FROM: Hacek DM, Bednarz P, Noskin GA, Zembower T, Peterson LR. Yield of vancomycin-resistant enterococci and multidrug-resistant Enterobacteriaceae from stools submitted for Clostridium difficile resting compared to results from a focused surveillance program. J Clin Microbiol 2001;39:1152-1154.

\section{Risk of Infection From Reused Virus-Contaminated Catheters}

Luijt and colleagues from the Regional Public Health Laboratory, Groningen, The Netherlands, conducted a study to determine the theoretical risk of virus transmission during reuse of catheters. An in vitro study was performed using an RNA virus (echovirus-11) and a DNA virus (adenovirus-2). After deliberate contamination of the catheters, reprocessing and reuse of the cleaned and glutaraldehyde-disinfected catheters was simulated. The presence of residual virus was determined by cell culture and by PCR. After the disinfection step, infectious enterovirus was detectable in one $(10 \%)$ of the samples, whereas two $(20 \%)$ contained detectable enterovirus RNA. After simulated reuse, a culture of enterovirus was obtained from one $(10 \%)$ of the catheters, but no less than six $(60 \%)$ of the samples were enterovirus PCR-positive, and one $(10 \%)$ contained detectable adenovirus DNA. After sonification of the catheter tips, no infectious virus could be detected, but enterovirus RNA was detected in two (20\%) and adenovirus DNA in three (30\%) of the samples.

The authors concluded that, even after rigorous cleaning and disinfection, virus was still present in the catheter. Reuse of catheters labeled for single-use only is dangerous and should be prevented. 
FROM: Luijt DS, Schirm J, Savelkoul PH, Hoekstra A. Risk of infection by reprocessed and resterilized viruscontaminated catheters: an in-vitro study. Eur Heart $J$ 2001;22:378-384.

\section{Ethylene Oxide Exposure Determinants}

LaMontagne and Kelsey, from the Dana-Farber Cancer Institute, Boston, conducted a study to identify determinants of workplace exposures to ethylene oxide to assess the effect OSHA's 1984 ethylene oxide standard. An in-depth survey of all hospitals in Massachusetts that used ethylene oxide from 1990 through 1992 (96\% participation, $\mathrm{n}=90$ ) was conducted. Three types of exposure events were modeled with logistic regression: exceeding the 8-hour action level, exceeding the 15-minute excursion limit, and worker exposures during unmeasured accidental releases. Covariates were drawn from data representing an ecologic framework including direct and indirect potential exposure determinants.

After adjustment for frequencies of ethylene oxide use and exposure monitoring, a significant inverse relation was observed between exceeding the action level and the use of combined sterilizer-aerators, an engineering control technology developed after the passage of the OSHA standard. Conversely, the use of positive-pressure sterilizers employing ethylene oxide gas mixtures was strongly related to both exceeding the excursion limit and the occurrence of accidental releases.

These findings provide evidence of a positive effect of OSHA's ethylene oxide standard and specific targets for future prevention and control efforts.

FROM: LaMontagne AD, Kelsey KT. Evaluating OSHA's ethylene oxide standard: exposure determinants in Massachusetts hospitals. Am J Public Health 2001;91: 412-417.

\section{Hand Washing, Gowns, and Gloves Reduce Pediatric Transplant Infections}

Nosocomial infection is an important contributor to morbidity and mortality in pediatric solid-organ transplantation. The relative effect of protective gown and glove isolation was compared with strict hand washing in pediatric intensive care unit (PICU) patients with solid-organ transplantation in a prospective randomized study.

Children in a 23-bed PICU with solid-organ transplantation were enrolled into a gown and glove protective isolation protocol or a strict handwashing protocol. Children with current infection or known exposure to varicella were excluded from the study initially or at readmission.

The infection rate in the overall PICU population did not change significantly from the year before the study compared to during the study (2.1/100 vs $1.95 / 100$ patientdays; $P=.4$ ) The infection rate in the gown-and-glove group (2.3/100 patient-days) was reduced significantly compared with the pre-study infection rate in the transplant popula- tion (4.9/100 patient-days; $P=.0008)$. Strict hand washing also significantly reduced the infection rate in the transplant population $(3.0 / 100$ patient-days; $P=.008)$. Compliance with gowning and gloving was $82 \%$ and compliance with hand washing was $76 \%$ (compared to $22 \%$ before study $[P<.0001]$ and $52 \%$ after the study $[P<.0001])$. Despite an increased mean length of stay in the PICU in the gown-and-glove group $(P=.014)$, there was a trend toward reduction in the incidence of infection in the gown-andglove group.

The authors concluded that increased compliance with hand washing was associated with a reduction in nosocomial infections, and gown-and-glove isolation appeared to have an additional protective effect. Thus, some nosocomial infections may be preventable in the pediatric solid-organ transplantation population.

FROM: Slota M, Green M, Farley A, Janosky J, Careillo J. The role of gown and glove isolation and strict handwashing in the reduction of nosocomial infection in children with solid organ transplantation. Crit Care Med 2001;29:405-412.

\section{Computerized Detection of Infections}

Rocha and colleagues, from the University of Utah, Salt Lake City, conducted a study to analyze whether computer-generated reminders about infections could influence clinicians' practice patterns and consequently improve the detection and management of nosocomial infections. The conclusions produced by an expert system developed to detect and manage infections were presented to the attending clinicians in a pediatric hospital to determine whether this information could improve detection and management. Clinician interventions were compared before and after the implementation of the system. The responses of the clinicians (staff physicians, physician assistants, and nurse practitioners) to the reminders were determined by review of paper medical charts. Main outcome measures were the number of suggestions to treat and manage infections that were followed before and after the implementation of COMPISS (Computerized Pediatric Infection Surveillance System). The clinicians' opinions about the system were assessed by means of a paper questionnaire distributed following the experiment.

The results failed to show a statistical difference between the clinicians' treatment strategies before and after implementation of the system $(P>33$ for clinicians working in the emergency room and $P .45$ for clinicians working in the pediatric intensive care unit). The questionnaire results showed that the respondents appreciated the information presented by the system.

The authors concluded that the computer-generated reminders about infections were unable to influence the practice patterns of clinicians, citing methodological problems as a possible contributing factor.

FROM: Rocha BH, Christenson JC, Evans RS, Gardner RM. Clinicians' response to computerized detection of infections. J Am Med Inform Assoc 2001;8:117-125. 